\title{
AARC Clinical Practice Guidelines: Phase 4
}

As we complete the first 20 years of the 21 st century, the words "evidence-based medicine" are indelibly etched into practice. What does this mean? This approach to practice is based on using the highest level of evidence. It means that we follow the science and not the opinions of experts, regardless of the prestige of those experts. There is always evidence, although often it exists only at a low level. The challenge is to develop the wisdom to distinguish between high-level evidence, with moderate to strong confidence, or lower-level evidence, with low confidence. Because the available evidence is daunting, systematic reviews and clinical practice guidelines (CPGs) increasingly are used to inform best practice.

The commitment of the American Association for Respiratory Care (AARC) to the development of CPGs dates back more than 30 years. ${ }^{1}$ In late 1989, incoming AARC President Jerome M. Sullivan PhD RRT FAARC charged me with the task to develop a process to write standards for the practice of respiratory therapy. A steering committee was created, and we quickly decided to focus on CPGs rather than standards. At that time, the development of CPGs was in its infancy; indeed, the AARC was one of the first professional organizations to publish CPGs.

The initial CPG steering committee was ambitious in its development of a standardized process for the CPGs that would be written. This was the first phase in the history of AARC CPGs. The members of the CPG steering committee formed subcommittees to develop CPGs on oxygen therapy, aerosol therapy, adult mechanical ventilation, diagnostics, neonatal/pediatrics, home care, and bronchial hygiene. The first 5 CPGs were published in the December 1991 issue of RESPIRATORy CARE, and 8 additional CPGs were published in August 1992. From 1991 to 1995,41 CPGs were published. From 1991 to 2013, 69 expert panel, reference-based CPGs (47 original, 22 revised) were published.

These early CPGs were not evidence-based - those words were not part of our vocabulary in the early 1990s. They were based on expert opinion. As self-proclaimed experts, we

Dr Hess has disclosed relationships with Ventec Life Systems, Daedalus Enterprises, Deadalus Enterprises, Jones and Bartlett, McGraw-Hill, and UpToDate.

Correspondence: Dean R Hess PhD RRT FAARC. E-mail: dhess@aarc.org. DOI: $10.4187 /$ respcare.08624 wrote the CPGs and then searched the literature for references to support what we wrote. This is the opposite of an evidence-based approach, wherein the literature search is done first, the quality of the evidence is assessed, and finally CPGs are written.

Although these early AARC CPGs were not evidencebased in the truest sense, they nonetheless had a tremendous impact on practice. They were widely regarded as one of the most important activities ever undertaken by the AARC. A successful respiratory therapy consult service based on the AARC CPGs was implemented. ${ }^{2}$ The implementation of local practice guidelines, based on an AARC CPG, reduced the incidence of adverse effects associated with endotracheal suction. ${ }^{3}$ The AARC CPGs were included in the National Guidelines Clearinghouse, giving them wide distribution.

By the end of the 20th century, it became clear that we needed to move toward CPGs that were evidence-based. The AARC's first evidence-based CPG was published in 2003. ${ }^{4}$ A second evidence-based CPG was published in 2010. ${ }^{5}$ This was the second phase of AARC CPGs. A lot was learned in creating these CPGs, not the least of which was the amount of effort required to bring these to completion. In the meantime, many of the originally published CPGs were becoming outdated.

From 2010 to 2013, 9 AARC guidelines were updated. In these CPGs, recommendations were written and evidence-based words were used (eg, "suggest" and "recommend"). ${ }^{6}$ Aside from that, the structure of the CPGs was similar to those published since the early 1990s.

It became increasingly recognized that the AARC should move beyond the development of expert panel, referencedbased CPGs in favor of evidence-based CPGs. To accomplish this, the AARC commissioned the Evidence-Based Practice Center at Vanderbilt University to assist with the development of evidence-based CPGs related to airway clearance. This led to the publication of $2 \mathrm{CPGs}^{7,8}$ and 2 systematic reviews. ${ }^{9,10}$ This represents the third phase of AARC CPGs. By the time of the publication of the airway clearance CPGs, the AARC had accumulated more than 20 years of experience writing and publishing guidelines.

This brings us to the fourth phase of AARC CPGs, the first of which are published in this issue of the Journal. ${ }^{11,12}$ This is an important step forward. These guidelines have many of the required elements of evidence-based CPGs. There is a PICO (patient, intervention, comparison, and outcome) question, 


\section{EDITORIALS}

which was the basis for a comprehensive literature search, the assessment and review of the available literature, and the writing of guidelines based on the available evidence.

The guideline recommendations for these CPGs combine the best available evidence with the collective experience of committee members. To achieve this, a modification of the RAND/UCLA Appropriateness Method was used. ${ }^{13}$ There was a clear attempt to avoid writing CPGs based on expert opinion. It is important to distinguish between experience and expert opinion. Experience is evidence, although at a low level. Opinion, on the other hand, is not evidence. To paraphrase the late Senator Daniel Patrick Moynihan, we are entitled to our own opinions, but not our own facts (eg, evidence). Important to guideline development, these CPGs were developed using an explicit methodology.

Note that CPGs are not intended as procedures and do not offer specific details about procedures. For example, the adult tracheostomy CPG recommends the use of a protocol to guide weaning and removal of the tracheostomy tube. ${ }^{11}$ The details are left to local practice; a standardized approach (protocol) might be more important than the specific details. The pediatric tracheostomy $\mathrm{CPG}^{12}$ recommends that tracheostomy tubes should be changed as needed secondary to obstruction, but the details of the tracheostomy tube change procedure are left to local practice. It is unrealistic to expect that any CPG will address the details of any procedure, as the available evidence is unlikely to ever have such granularity.

In summary, the AARC has a long history of publishing CPGs. This latest phase of CPG development is a major step forward in the era of evidence-based medicine. We anxiously await more AARC CPGs using this model to inform respiratory care practice.

Dean R Hess

Managing Editor, RESPIRATORY CARE Massachusetts General Hospital Northeastern University Boston, Massachusetts

\section{REFERENCES}

1. Hess D. The AARC clinical practice guidelines. Respir Care 1991;36 (12):1398-1401

2. Stoller JK, Mascha EJ, Kester L, Haney D. Randomized controlled trial of physician-directed versus respiratory therapy consult servicedirected respiratory care to adult non-ICU inpatients. Am J Respir Crit Care Med 1998;158(4):1068-1075.

3. Maggiore SM, Lellouche F, Pignataro C, Girou E, Maitre B, Richard JC, et al. Decreasing the adverse effects of endotracheal suctioning during mechanical ventilation by changing practice. Respir Care 2013;58 (10):1588-1597.

4. Hess DR, Kallstrom TJ, Mottram CD, Myers TR, Sorenson HM, Vines DL. Care of the Ventilator Circuit and Its Relation to Ventilator-Associated Pneumonia. Respir Care 2003;48(9):869-879.

5. DiBlasi RM, Myers TR, Hess DR. Evidence-Based Clinical Practice Guideline: Inhaled Nitric Oxide for Neonates With Acute Hypoxic Respiratory Failure. Respir Care 2010;55(12):1717-1745.

6. Restrepo RA. Clinical practice guidelines: from "reference-based" to "evidence-based". Respir Care 2010;55(6):787-789.

7. Strickland SL, Rubin BK, Drescher GS, Haas CF, O'Malley CA, Volsko TA, et al. AARC Clinical Practice Guideline: effectiveness of nonpharmacologic airway clearance therapies in hospitalized patients. Respir Care 2013;58(12):2187-2193.

8. Strickland SL, Rubin BK, Haas CF, Volsko TA, Drescher GS, O'Malley CA. AARC Clinical Practice Guideline: effectiveness of pharmacologic airway clearance therapies in hospitalized patients. Respir Care 2015;60(7):1071-1077.

9. Andrews J, Sathe NA, Krishnaswami S, McPheeters ML. Nonpharmacologic airway clearance techniques in hospitalized patients: a systematic review. Respir Care 2013;58(12):2160-2186.

10. Sathe NA, Krishnaswami S, Andrews J, Ficzere C, McPheeters ML. Pharmacologic agents that promote airway clearance in hospitalized subjects: a systematic review. Respir Care 2015;60(7):1061-1070.

11. Mussa CC, Gomaa D, Rowley DD, Schmidt U, Ginier E, Strickland SL. AARC clinical practice guideline: management of adult patients with tracheostomy in the acute care setting. Respir Care 2021;66 (1):156-169.

12. Volsko TA, Parker SW, Deakins K, Walsh BK, Fedor KL, Valika T, et al. AARC Clinical Practice Guideline: management of pediatric patients with tracheostomy in the acute care setting. Respir Care 2021;66 (1):144-159.

13. Fitch K, Bernstein SJ, Aguilar MD, Burnand B, LaCalle JR, Lazaro P, et al. The RAND/UCLA Appropriateness Method User's Manual. Santa Monica, CA: RAND; 2001. 